\title{
VALIDATION OF THE LARGE INTERFACE METHOD OF NEPTUNE_CFD 1.0.8 FOR PRESSURIZED THERMAL SHOCK (PTS) APPLICATIONS
}

\author{
P. Coste ${ }^{1}$, J. Laviéville ${ }^{2}$, J. Pouvreau ${ }^{l}$, C. Baudry ${ }^{2}$, M. Guingo ${ }^{2}$, A. Douce ${ }^{2}$ \\ ${ }^{I}$ Commissariat à l'Energie Atomique, Grenoble, FRANCE.pierre.coste@cea.fr \\ ${ }^{2}$ Electricité de France, Chatou, FRANCE.
}

\begin{abstract}
NEPTUNE_CFD is a code based on a 3D transient Eulerian two-fluid model. One of the main application targets is the two-phase Pressurized Thermal Shock (PTS), which is related to PWR Reactor Pressure Vessel (RPV) lifetime safety studies, when sub-cooled water from Emergency Core Cooling (ECC) system is injected into the possibly uncovered cold leg and penetrates into the RPV downcomer. Five experiments were selected for the validation. A co-current smooth and wavy airwater stratified flow in a rectangular channel with detailed measurements of turbulence and velocities allows to validate the dynamic models. A co-current smooth and wavy steam-water stratified flow in a rectangular channel with measurements of the steam flow rates allows to validate the condensation models. Two experiments dealing with a water jet impingement on a water pool free surface in air environment allow to validate the dynamic models in the situation of a jet impinging a pool free surface. Finally, the COSI experiment allows to validate all the models involved in the reactor conditions. The calculations are done with the same set of Large Interface Method (LIM) models and a RANS (k- $\varepsilon$ ) approach for turbulence. They substantiate the application of the tool to PTS studies.
\end{abstract}

\section{INTRODUCTION}

One of the main application targets of two-phase CFD for Nuclear Reactor Safety (NRS) in France is the two-phase Pressurized Thermal Shock (PTS), which is related to PWR Reactor Pressure Vessel (RPV) lifetime safety studies, when sub-cooled water from Emergency Core Cooling (ECC) system is injected into the possibly uncovered cold leg, and penetrates into the RPV downcomer. Following the NEA/CSNI Best Practice Guidelines (BPG's), relevant PTS-scenarios have been identified. A Phenomena Identification and Ranking Table (PIRT) process, the related state of the art of modeling and the existing data basis have been reviewed by a panel of European experts, mainly within the ECORA and NURESIM projects (Lucas et al., 2009). In the situations of interest, the cold leg is thought to be a liquid-vapor stratified flow; the ECC jet is thought not to break-up and to plunge into the free surface; direct contact condensation takes place with a maximum in the ECC region of the cold leg; the free surfaces are thought to be mostly wavy or rough; the water and gas are turbulent.

Consistently, the following five experiments were selected for the CFD validation dedicated to PTS presented in this paper. The first four are useful for separate effects validation. The Fabre et al. (1987) experiment is a co-current smooth and wavy Air Water STratified (AWST) flow in a rectangular channel with detailed measurements of turbulence and velocities. The Lim et al. (1984) experiment is a co-current smooth and wavy Steam Water STratified (SWST) flow in a rectangular channel with measurements of the steam flow rates at six axial positions along the channel. The Bonetto and Lahey (1993) and the Iguchi et al. (1998) experiments deal with a water jet impingement on a water pool free surface in air environment. In the first one, the void fraction and the mean velocities are measured whereas in the second one, mean and rms velocities are measured. Finally, the COSI experiment represents a cold leg scaled 1/100 for volume and power from a 900 MW PWR under LOCA conditions, and therefore can be used for global validation. The measurements include temperature profiles at eight axial positions in the pipe and global condensation rates, at various ECC flow rates, inlet steam flow rates and water level in the cold leg. As COSI is the closest test to the final application, as the combination of the separate effects is unknown, as separate effects tests cover 
separate phenomena involved in the final application with various discrepancies, the expert judgment of the validation with respect to the PTS application is built putting the highest weight on the COSI test.

The calculations presented in this paper are done with the tool mainly devoted to two-phase CFD for NRS in France: NEPTUNE_CFD (NCFD), based on a 3D transient Eulerian two-fluid model (Méchitoua et al. (2003), Guelfi et al. (2007)). They are all done with the same version 1.0.8, with the same set of models, which includes the Large Interface Method (LIM) and a RANS approach with (k$\varepsilon)$ transport equations in each phase. Following the BPGs, the mesh sensitivity is investigated.

The purpose of this paper is to present the validation of NCFD 1.0.8, not to give details on the modeling: therefore in section 2, only the main principles of the modeling are described. Then the next sections cover the five test cases.

\section{MODEL DESCRIPTION}

\subsection{Two-phase model and solver}

For our CFD approach of the PTS problem, the two-fluid model was selected because of its generality and its intrinsic ability to treat heat and mass transfers at large scales, at any Reynolds numbers and more generally in a wide range of thermohydraulics conditions. The six transport equations (mass, momentum and energy for both liquid and gas) of the two-phase model, with the same pressure in the two phases, as established for example in Ishii (1975), are solved.

The Reynolds stress tensor is closed with the Boussinesq eddy viscosity assumption. A twoequation k- $\varepsilon$ model for the calculation of the turbulent eddy viscosity is used: one for each phase in the whole domain. It is an extension to multi-phase flows of the classical model used in single phase flows. The decrease of turbulence due to thermal stratification is included. The turbulence production due to the influence of each phase on the other one depends along the LI's on the interfacial friction.

The NCFD solver is based on a pressure correction fractional step approach. The discretization follows a 3D full unstructured finite volume approach, with a collocated arrangement of all variables. Numerical consistency and precision for diffusive and advective fluxes for non-orthogonal and irregular cells are taken into account through a gradient reconstruction technique. Convective schemes for all variables, except pressure, are centered/upwind scheme. Velocities components can be computed with a full centered scheme. Gradients are calculated at second order for regular cells and at first order for highly irregular cells. The time step is variable with a CFL condition generally equal to unity.

\subsection{Large Interface Method}

The two-phase model resulting from averaging process leads to specific problems in case of flows with free surfaces like stratified flows or liquid jets in gas. In CFD, such flows lead to interfaces much larger than cells size, called afterward Large Interfaces (LI's). In this case, specific closure laws are needed in the LI regions which are different from the closure laws outside. It is then necessary to locate the LI's position at each time step of the simulation in order to apply the correct closure laws. Our approach differs from approaches which aim at simulating the LI's location in the context of one momentum equation, like Volume Of Fluid, level set or front tracking, in that it only locates the position, it does not reconstruct it. In the calculations of this paper, the surface tension on LI's is not directly numerically simulated because it plays a role at length scales which are generally much smaller than the ones simulated by our CFD.

The interface detection method implemented in NCFD is based on the gradient of liquid fraction. The first step consists in computing a refined liquid fraction gradient, based on harmonic or antiharmonic interpolated values of liquid fraction on the faces between the cells (Laviéville and Coste, 2008). This refined gradient allows to detect the cells belonging to the LI.

The specific LI's closure laws developed and implemented in NCFD (Coste et al., 2007, 2008, 2009) consider not only the cells crossed by the LI but also the two neighboring cells located in LI's normal direction, on both sides, liquid and vapor: the considered LI is three-cell thick (LI3C). It 
allows to use only physically relevant values by choosing the interface side where the phase is not residual. It allows also to limit the effect of the LI's position with regard to the meshing.

\subsection{Friction}

An anisotropic two-phase friction is assumed close to the free surfaces. This is because the friction of bubbles with liquid, in the case of bubbles coming up to a free horizontal stratified flow surface, for example, is different from the friction of the gas over the free surface, which sees the liquid rather like a wall. Then the drag coefficient in the direction normal to the LI is a general two-phase one (derived from bubbles or drops drags) whereas the drag coefficient in the LI plane is specific to LI's. The basic hypothesis is that the free surface is a wall for the gas, a wall moving at the interface velocity $u_{\text {int }}$, a wall that can be rough when there are waves.

\subsection{Heat and mass transfer}

The important closure law for direct contact condensation is the heat transfer coefficient $h_{L, i}$ between the liquid and the interface. There are many models available since several decades (Theofanous et al., 1976) (Banerjee, 1978) (Bankoff, 1980). They are generally not directly suitable for CFD in which the models use local variables available in each computation cell. The model currently used in NCFD in case of wavy flows (Coste and Laviéville, 2009) is a wall law form of the model originally tested in the SIMMER code (Coste, 2004) in which it could predict the trends of the COSI tests without fitting constants. Its form comes from the surface renewal model with a renewal frequency built with the Kolmogorov length and the turbulent velocity. Recently, DNS and LES brought new insights and clarifications (Magnaudet and Calmet, 2006). DNS of Lakehal et al. (2007) showed that the surface divergence model of Banerjee et al. (2004) coming from the Hunt and Graham theory applies in the liquid phase. The model currently used in NCFD in case of smooth flows is a wall law form of this model.

\section{AIR-WATER STRATIFIED FLOW}

\subsection{Description of the test case}

We consider an experiment featuring an adiabatic air water co-current stratified flow, in a $12 \mathrm{~m}$ long, $20 \mathrm{~cm}$ wide, $10 \mathrm{~cm}$ high, $0.1 \%$ slope rectangular channel (Fabre et al., 1987). The inlet liquid superficial velocity is $0.15 \mathrm{~m} / \mathrm{s}$, which corresponds to a water flow rate equal to $31 / \mathrm{s}$. Our selected tests are the runs 250 and 400 . The inlet gas superficial velocity of run $250,2.5 \mathrm{~m} / \mathrm{s}$, is high enough for the interfacial friction to play an important role, and sufficiently weak for waves to be negligible. The inlet gas superficial velocity of run $400,4.0 \mathrm{~m} / \mathrm{s}$, is high enough for waves and 3D circulations. In both runs, the Reynolds numbers (19000-31000) are large enough to validate the use of the turbulence model. The water height is $3.8 \mathrm{~cm}$ (resp. 3.15) in the run 250 (resp. 400). The measurements used for the CFD comparisons are done $9.1 \mathrm{~m}$ from the inlet, on a vertical line containing the channel axis. These are vertical profiles of volume fraction, average velocities and turbulence quantities, in liquid and gas.

\subsection{Computational representation}

The friction along lateral walls leads us to consider 3D-meshes for the calculations. They are uniform, structured and rectangular. Three different refined meshes (coarse, intermediate and fine) are used, in order to check the results sensibility to the mesh size, with $\left(125^{*} 10 * 10\right),(250 * 20 * 20)$, $(500 * 40 * 40)$ cells in length, height and depth. The three directions are $(x, y, z): \mathrm{x}$ is the channel axis, axial, $\mathrm{y}$ is the height, $\mathrm{z}$ is the third direction. The comparisons between calculations and experiment are in Fig. 1 and Fig. 2. The most important parameters from the PTS point of view are the horizontal average velocities $u_{x}$ (horiz. velocity) and $k$ (turb. kin. energy) on the liquid (liq.) side $\left(u_{L, x}, k_{L}\right)$, in particular close to the free surface. On the gas side close to the free surface, the average horizontal 
velocity $u_{G, x}$ is important because it has a strong effect on the interfacial friction, which in turn has an effect on $k_{L}$ close to the surface.

\subsection{Results}

The Fig. 1 and Fig. 2 show the calculations results and their comparisons with the experimental data $9.1 \mathrm{~m}$ from the inlet, of runs 250 and 400 . The left (resp, right) graphs present liquid (resp. gas) dynamics; the top (resp. bottom) graphs show the mean velocity (resp. turbulent kinetic energy); the xaxis is directed from the wall to the interface, so the liquid-gas interface is always located on the right of the graphs. The calculation results (lines) are compared with the experiment (dark symbols); the black, red and green lines denote resp. the coarse, intermediate and finest meshes CFD results.

The Fig. 1 shows the calculations of Run 250 (smooth free surface). The calculated liquid axial horizontal velocity profile does not have the measured S-like shape due to the liquid entrainment by the gas just below the free surface: a feature of the flow is missing; nevertheless the liquid velocity profile and the turbulent level are correctly predicted. The liquid turbulent shear stress close to the wall and close to the free surface has some discrepancies with the measurements, which is probably caused by the use of a high-Reynolds turbulence model. The gas velocity and turbulent kinetic energy are correctly predicted, but the finest mesh seems to over-estimate turbulence near the interface. Eventually the agreement and the weak sensitivity to the mesh refinement are satisfactory for a CFD use in PTS, at the present stage.

The Fig. 2 shows the NCFD calculations of run 400, a run with a wavy free surface. The discrepancy between the experimental results and the calculation is larger. The effects of waves are clear: the liquid velocity S-like shape is more accentuated and the turbulent kinetic energy is largely above the interface. Near the interface, the liquid velocity is correctly predicted while the turbulent kinetic energy is under-predicted using coarse meshes.

Correct results of such wavy flows as Run 400 were obtained by (Meknassi et al., 2000) or (Berthelsen and Ytrehus, 2005), with a steady state and fully developed mean flow approach. Meknassi et al. (2000) used a $k-\varepsilon$ model and algebraic closures to model secondary flows controlled by the anisotropy of turbulence. In its present stage, (transient and 3D) NCFD does not take them into account and then can not predict Run 400-like flows as well as those more specialized (steady state and fully developed mean channel flow) tools. 

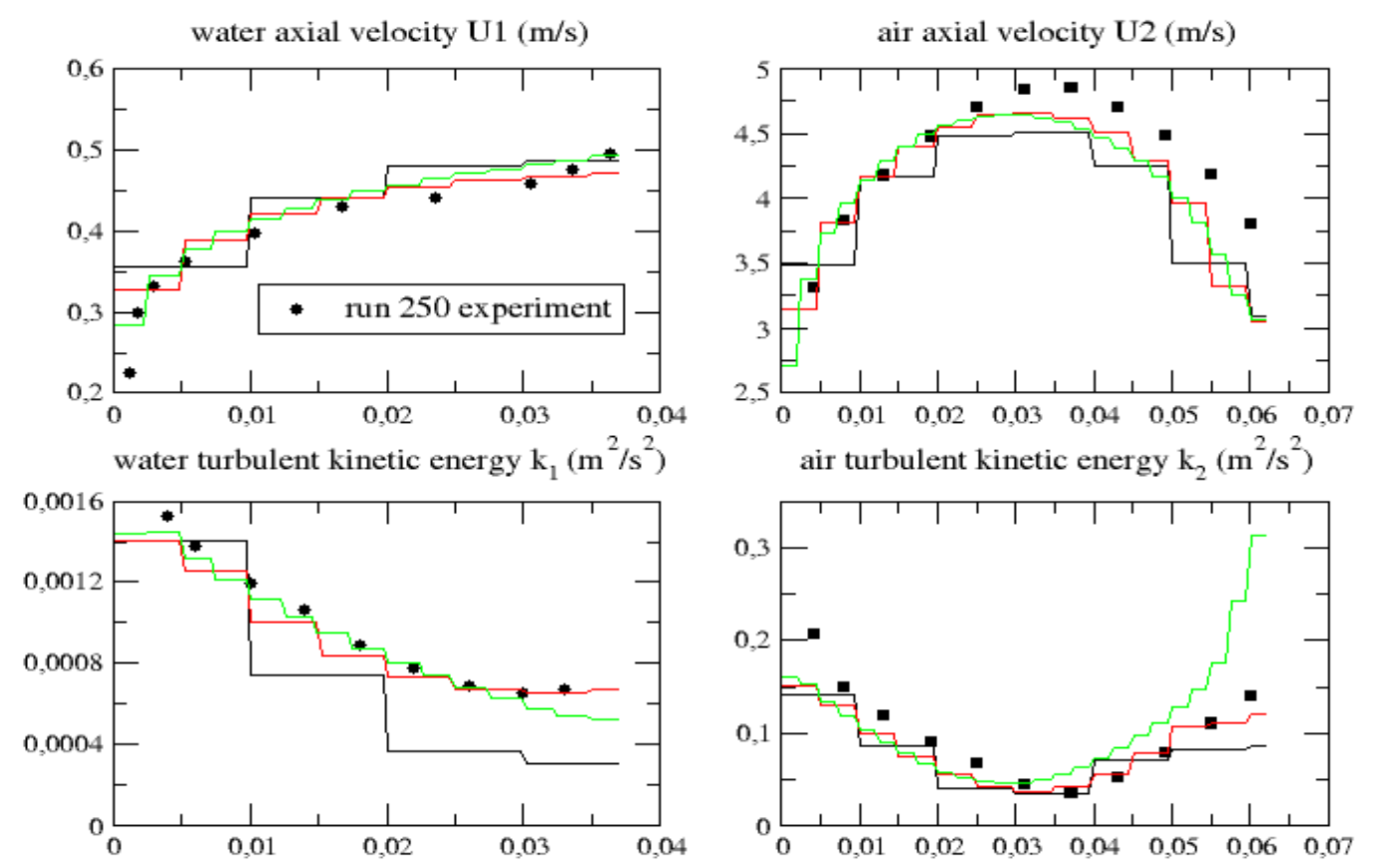

Fig. 1 : Run 250 (smooth) - NCFD Calculation with large interface turbulence and friction models with the coarse mesh (-), intermediate mesh (-), finest mesh (-) versus measurements (symbols).
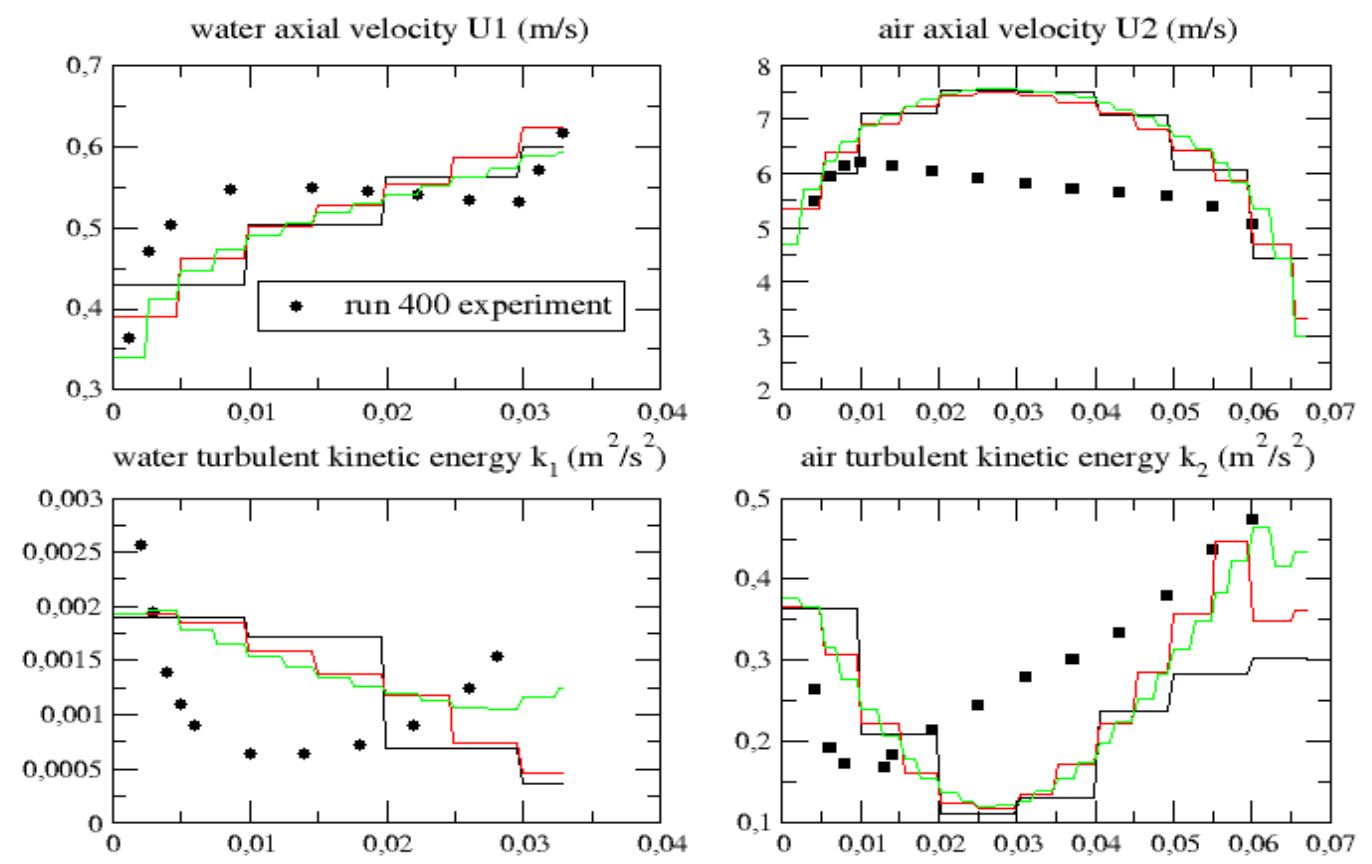

Fig. 2 : Run 400 (wavy) - NCFD Calculation with large interface turbulence and friction models with the coarse mesh (-), intermediate mesh (-), finest mesh (-) versus measurements (symbols). 


\section{STEAM WATER STRATIFIED FLOW}

\subsection{Description of the test case}

The experimental data are from Lim et al. (1984). They deal with a Steam-Water co-current STratified flow (SWST) in a rectangular channel. The flow regime of the free surface was observed: smooth, transition or wavy. Those runs were calculated by Yao et al. (2005) who found that no CFD model was able to satisfactorily calculate both smooth and wavy runs. Now the more recent model implemented in NCFD aims at covering both regimes.

The test section is a rectangular channel. Its dimensions are: $6.35 \mathrm{~cm}$ high, $l=30.48 \mathrm{~cm}$ wide, 160.1 $\mathrm{cm}$ long. It is designed to be two-dimensional. The inlet water height is $\delta_{L}=1.59 \mathrm{~cm}$. The selected test cases are listed in Table 1. For each case, the measured quantities used for the inlet boundary conditions (BC's) are the liquid and gas mass flow rates $\dot{m}_{L}, \dot{m}_{G}$, and temperatures $T_{L}, T_{G}$. The outlet pressure is the atmosphere. The inlet liquid temperature is $25^{\circ} \mathrm{C}$.

\begin{tabular}{|c|c|c|c|c|c|c|c|c|}
\hline Case & $\begin{array}{l}\text { Free } \\
\text { surface }\end{array}$ & $\begin{array}{l}\dot{m}_{L} \\
(\mathbf{k g} / \mathbf{s})\end{array}$ & $\begin{array}{l}\dot{m}_{G} \\
(\mathbf{k g} / \mathbf{s})\end{array}$ & $\begin{array}{l}T_{G} \\
\left({ }^{\circ} \mathbf{C}\right)\end{array}$ & $\begin{array}{l}k_{L} \\
\left(\mathbf{m}^{2} \cdot \mathbf{s}^{-2}\right)\end{array}$ & $\begin{array}{l}k_{G} \\
\left(\mathrm{~m}^{2} \cdot \mathrm{s}^{-2}\right)\end{array}$ & $\begin{array}{l}\mathcal{E}_{L} \\
\left(\mathrm{~m}^{2} \cdot \mathbf{s}^{-3}\right)\end{array}$ & $\begin{array}{l}\mathcal{E}_{G} \\
\left(\mathbf{m}^{2} \cdot \mathbf{s}^{-3}\right)\end{array}$ \\
\hline 1 & $\mathrm{~S}$ & 0.657 & 0.041 & 111 & $2.810^{-4}$ & 0.36 & $3.910^{-4}$ & 6.1 \\
\hline 2 & S-W & 0.657 & 0.065 & 116 & $2.810^{-4}$ & 0.92 & $3.910^{-4}$ & 24 \\
\hline 4 & S-W & 0.657 & 0.124 & 126 & $2.810^{-4}$ & 3.3 & $3.910^{-4}$ & 170 \\
\hline 8 & W & 1.44 & 0.126 & 125 & $1.310^{-3}$ & 3.4 & $4.110^{-3}$ & 180 \\
\hline
\end{tabular}

Table 1 : Lim et al. (1984) runs used for present validation. k- $\varepsilon$ values: estimated turbulent inlet boundary conditions for our calculations.

\subsection{Computational representation}

The experiment geometry and our RANS modeling approach for turbulence allow to use a $2 \mathrm{D}$ computation. Three orthogonal conform meshes have been used, $\mathrm{m}_{2}$ (7698 nodes, 18 in the vertical direction that is about 8 in the liquid layer), $\mathrm{m}_{4}$ (30114 nodes), $\mathrm{m}_{8}$ (119106 nodes), $\mathrm{m}_{2 \mathrm{~N}}$ being obtained from $m_{N}$ by multiplying the number of cells in each spatial direction by 2 . The inlet BC's for turbulent quantities $k$ and $\varepsilon$ (Table 1) are estimated orders of magnitude. Their sensitivity was tested to a certain extent in a previous study (Coste and Laviéville, 2009).

\subsection{Results}

In the SWST1 run (Fig. 3, top left), the free surface was observed as smooth. The calculations are unsatisfactorily sensitive to the mesh. It is mostly problematic in this run and less in the others. With the rough mesh $\mathrm{m}_{2}$, the flow is detected as smooth and then the calculation is more or less correct. With the intermediate mesh $\mathrm{m}_{4}$, for a reason that has to be investigated in the future, the liquid TKE is higher, so the flow is detected as in transition between $0.5 \mathrm{~m}$ and $0.8 \mathrm{~m}$ from the inlet: the result is then sensitive to the mesh and there is an overestimation of the condensation in this region of the channel. With the fine mesh $\mathrm{m}_{8}$, for probably the same reason, the liquid TKE is even higher, as soon as the liquid flows from the inlet, so the flow is wrongly detected as in transition or even as wavy, leading to a large overestimation of the condensation. Closely downstream of the inlet, a kind of wave takes place. This wave goes with an increase of the liquid TKE close to the free surface whereas neither such wave nor TKE increase takes place with $\mathrm{m}_{2}$. The TKE increase implies an automatic detection by the heat transfer model of a wavy free surface, which then switches to an increase of heat transfer from the liquid to the interface, leading to a higher condensation rate. On the other hand, with $\mathrm{m}_{2}$, the free surface is detected as smooth, which leads to a lower condensation rate. As the difference takes place closely downstream of the inlet, additional sensitivity calculations about BCs with $\mathrm{m}_{8}$ were done. In the region closely downstream of the inlet, the sensitivity on the liquid TKE was high but looking on the whole test section, the consequences on the axial vapor mass flow rates were 
negligible. It is therefore not encouraging to look for a solution of the problem just by adjusting the BC's. The present thinking goes rather towards the LIM and $k$ - $\varepsilon$ modeling.

In the SWST2 run (Fig. 3, top right), the free surface measurements showed that it was smooth up to about $40 \mathrm{~cm}$ downstream of the inlet and then it turned to wavy. As in the SWST1 case, the rough mesh calculation better corresponds to this but the results are more satisfactory than SWST1 as the sensitivity to the mesh is more reasonable.

The SWST4 run (Fig. 3, bottom left) is also an intermediate run between smooth and wavy. The sensitivity to the mesh is again too large and requires further study.

The SWST8 run (Fig. 3, bottom right) is wavy. The results and the sensitivity to the mesh are more satisfactory than the runs where smooth free surfaces were observed. The increase of condensation due to the increase of the inlet liquid velocity in comparison with SWST4 is predicted, which is satisfactory.

As long as no measurement of both condensation and turbulence close to the free surface are not available, the further analysis will remain difficult as the errors of turbulence modeling on the one hand and the errors of heat and mass transfer modeling on the other hand will remain unclearly juxtaposed. However the results are improved in comparison with previous models which did not include the difference between modeling smooth and wavy flows (Yao et al., 2005).
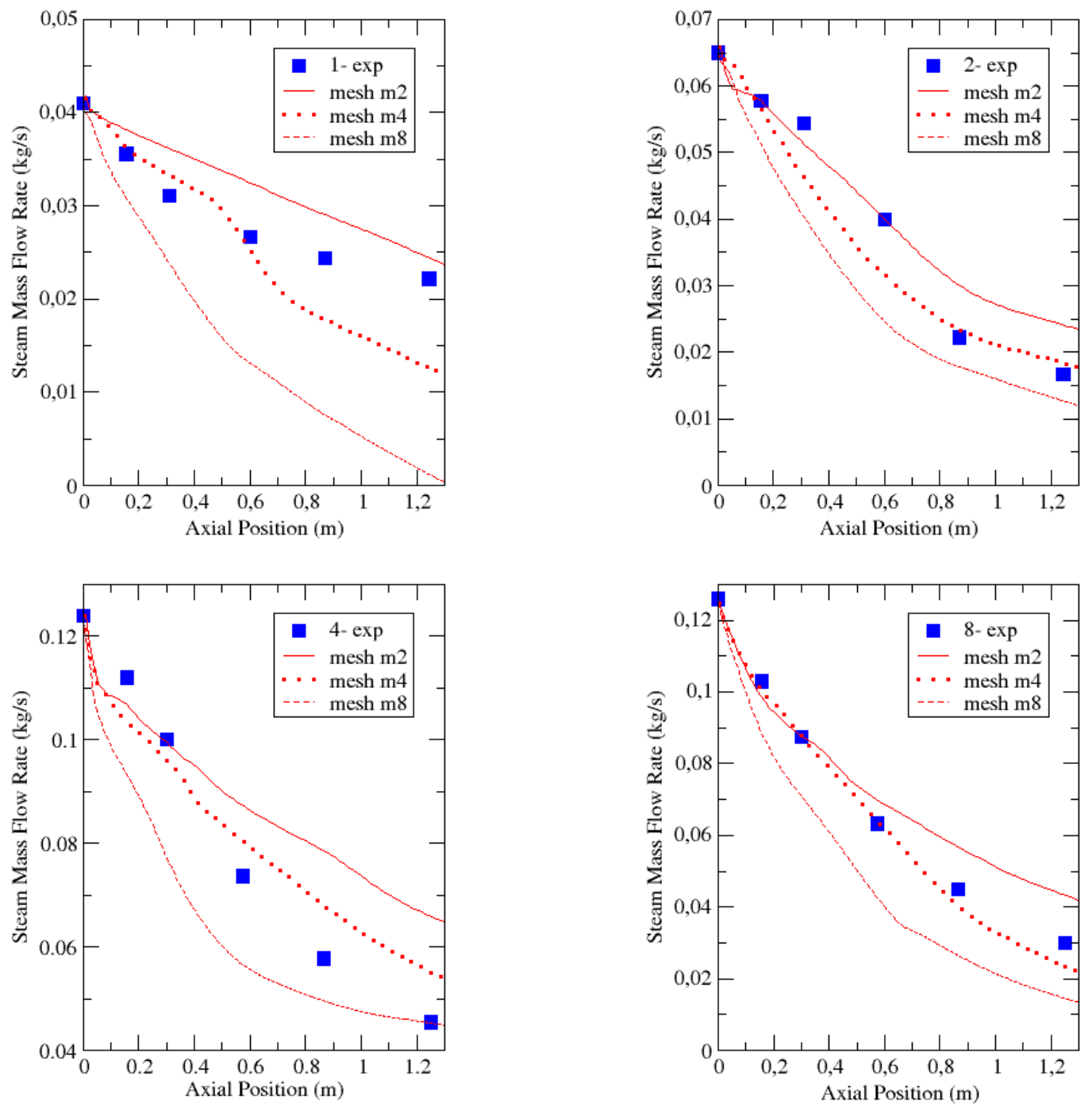

Fig. 3 NCFD calculations of SWST with the three meshes $\mathrm{m} 2, \mathrm{~m} 4$ and $\mathrm{m} 8$. Comparisons with the measured axial vapor flow profiles. Top left : SWST1, smooth. Top right: SWST2, intermediate.

Bottom left: SWST4, wavy. Bottom right: SWST8, wavy. 


\section{AIR ENTRAINMENT BY A PLUNGING LIQUID JET}

\subsection{Description of the test case}

Jet impingement on free surface may occur in the PWR's cold legs, in the PTS scenarios (Lucas et al., 2009) where a stratified flow in the cold legs takes place, with a low enough liquid height, when ECC water is injected. The impinging jet flow on a free surface is a particularly challenging case for multiphase models. The process of steam carry under and subsequent bubble dispersion (both by liquid and bubble induced turbulence), has to be captured by the multiphase models.

Bonetto and Lahey (1993) investigated in an air environment the liquid water jet impingement on a free surface. The axisymmetric and turbulent jet is impinging orthogonally on the free surface. In the experimental run selected for our calculation, the height of the nozzle above the undisturbed surface is $30 \mathrm{~mm}$. When a threshold velocity is exceeded, it is observed that the plunging liquid jet causes air entrainment. The flow is statistically steady-state. The void fraction radial profiles are measured at varying depths from the undisturbed surface. The liquid and gas velocities are measured for a single nozzle height. Different two-phase jet characteristics are noticed, depending on the turbulence intensity of the plunging liquid jet. The turbulence intensity of the liquid is varied to obtain a smooth jet (intensity 0.8 ) and a rough jet (intensity 3 ). For the first jet, the diameters of the induced bubbles were in the range $15-300 \mu \mathrm{m}$. For the second jet, a mean bubble size of $2 \mathrm{~mm}$ is measured. We compute this last.

\subsection{Computational representation}

The experiment geometry and our RANS modeling approach for turbulence allow to use a 2D axisymmetric computation. Three orthogonal conform meshes have been used, $\mathrm{m} 1$ (2333 nodes), $\mathrm{m} 2$ (10039 nodes), $\mathrm{m} 3$ (41597 nodes). The flow is two-phase, isothermal and turbulent. The gravity is taken into account. The densities are constant. We consider the $k-\varepsilon$ turbulence model for water.

\subsection{Results}

The physical steady state is reached by a transient calculation of $20 \mathrm{~s}$. Further $10 \mathrm{~s}$ of calculation are done in order to get the average values of void fraction, liquid and gas velocities which are presented here. The instantaneous values would not have been meaningful because the computed fields are not stationary.

In Fig. 4, the calculated radial profiles of the void fraction at three different depths below the undisturbed surface are compared with the measurements. The calculations are done with the three meshes which gives the sensitivity to the mesh. The coarser mesh profiles at $1 \mathrm{~mm}$ and $18 \mathrm{~mm}$ are very different from the ones obtained with the two finer meshes. An under-prediction of the air entrainment along the symmetry axis is calculated. Furthermore, the entrained air plum length is under-predicted.

The Fig. 5 shows the sensitivity to the mesh in relation to both the void fraction and the turbulent energy. When the mesh is refined, the liquid turbulent kinetic energy increases whereas the entrained air plum length decreases.

In the present state of the art, the CFD does not predict satisfactorily such flows. Schmidkte and Lucas (2009) proposed a model in CFX-11 with two parameters that could be adjusted in the future. As summarized by Davoust et al. (2002), there are two complementary mechanisms that drive the air entrainment: 1) the friction along the jet that brings down the air, and 2) the entrapment process at the point of impact. The mechanism (1) may be predicted more or less correctly by models dedicated to LI's as described in section 2.3. The mechanism (2) is much more problematic, as it generally takes place at intermediate length scales between simulation and modeling, a vague zone seldom explored in the literature which requires specific approaches (Pigny and Coste, 2005). 


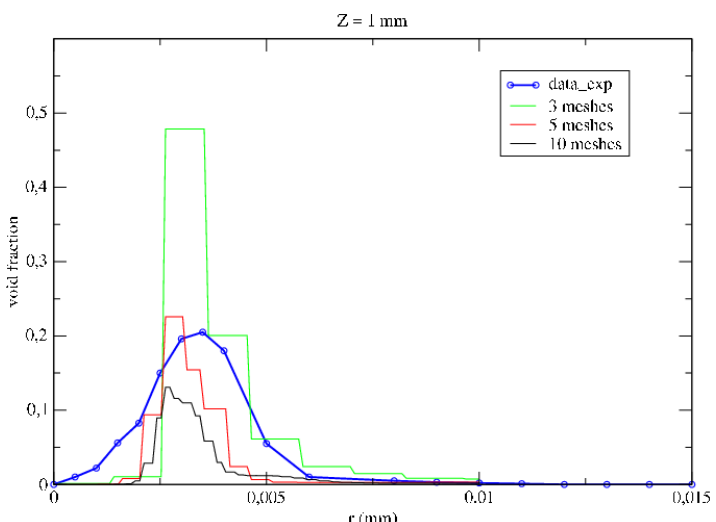

(a)

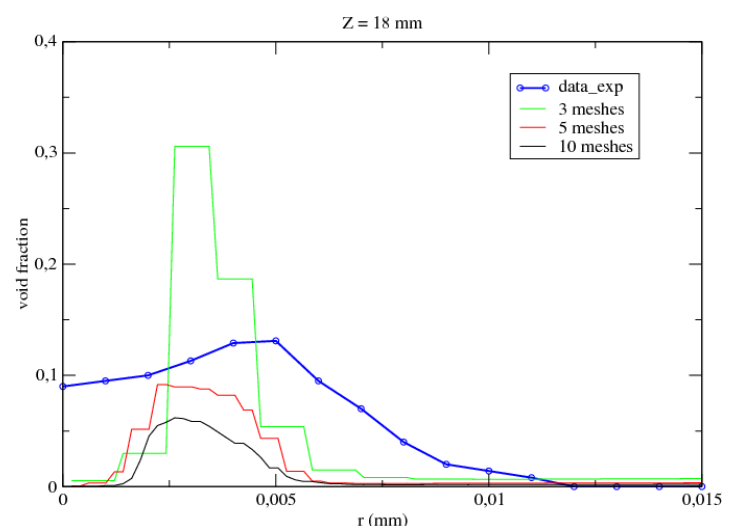

(b)

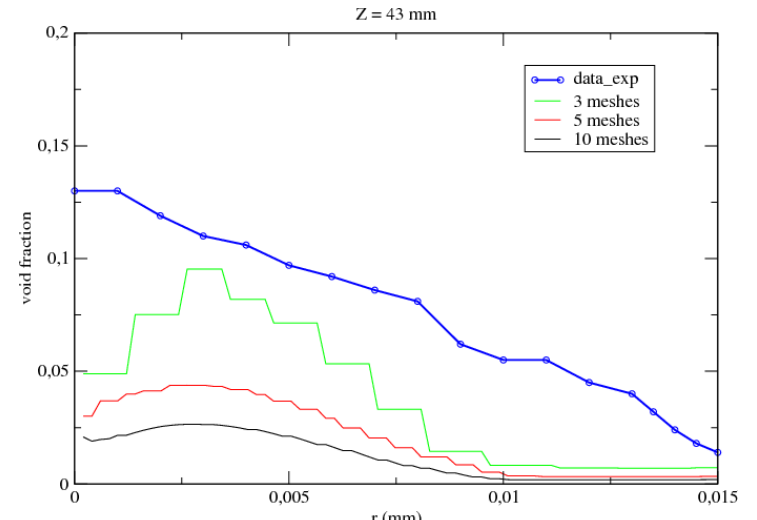

(c)

Fig. 4 : Calculated and measured void fraction radial profiles at three different depths below the undisturbed surface: $1 \mathrm{~mm}(\mathrm{a}), 18 \mathrm{~mm}(\mathrm{~b})$ and $43 \mathrm{~mm}$.

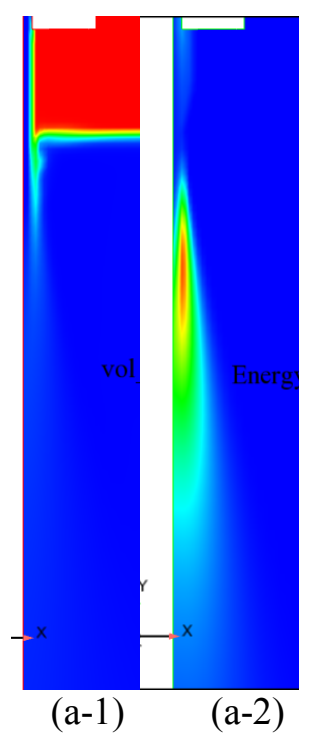

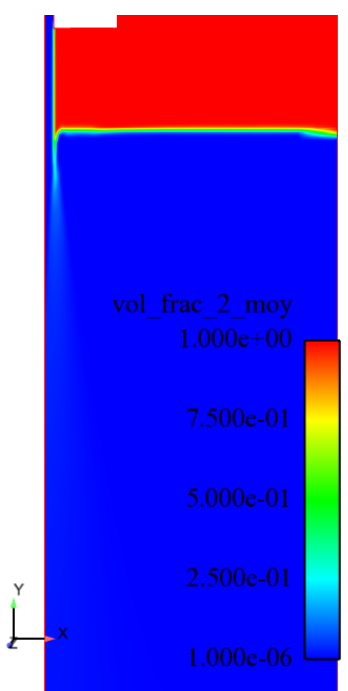

(b-1)

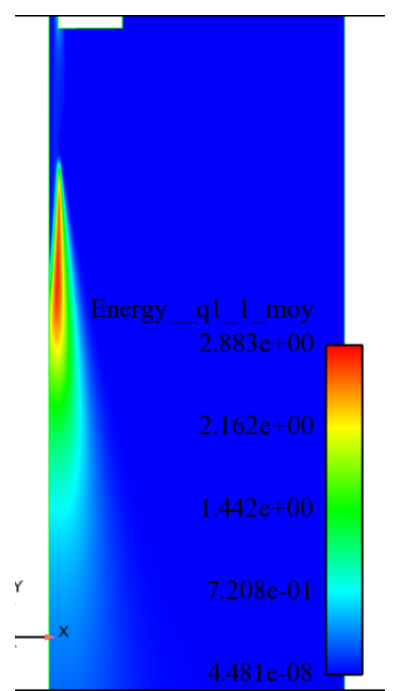

(b-2)

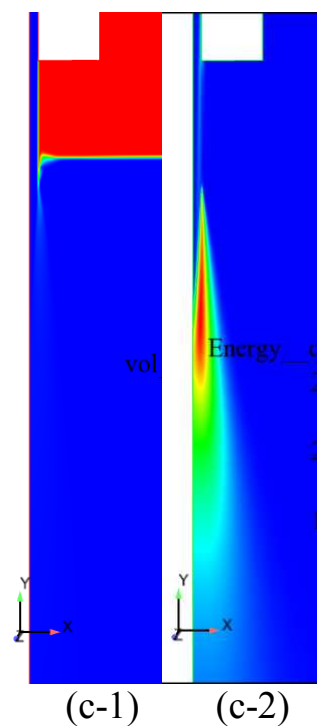

(c-1)

(c-2)

Fig. 5 : Calculated average void fraction (figures (-1), scale on (b-1)) and average liquid turbulent kinetic energy (figures (-2), scale on (b-2)) with three meshes: rough $\mathrm{m} 1$ (figures a-), intermediate $\mathrm{m} 2$ (b-) and finer $\mathrm{m} 3$ (c-). The views (a-) and (c-) show only the most interesting part of the computed domain. 


\section{DYNAMICS BELOW A PLUNGING LIQUID JET}

\subsection{Description of the test case}

The experimental data are from Igushi et al. (1998). They investigated the jet influence on a free surface and the effect of gas entrainment on liquid velocity and turbulence characteristics, using an axisymmetric, turbulent jet impinging orthogonally a free surface, in a cylindrical vessel (diameter $=$ $20 \mathrm{~cm}$, height $=39 \mathrm{~cm}$ ). The flow reaches a statistically steady-state; the fluid is water in an air environment. In the experiment, the liquid mean and rms velocities are measured for different heights of the nozzle above the undisturbed surface at varying depths from the surface and radial distance. When the nozzle height is long enough, the jet surface is rough and large bubbles are generated, the dispersion region being localized beneath the pipe exit. In this configuration the structure of velocity and turbulence beyond the surface is modified, comparing with a single-phase jet. This study has investigated this pattern, with a liquid flow rate equal to $50 \mathrm{~cm}^{3} / \mathrm{s}$ and a nozzle height equal to $1 \mathrm{~cm}$; the internal diameter of the circular injection pipe is equal to $5 \mathrm{~mm}$.

\subsection{Computational representation}

The computational domain has been optimized in the radial direction without influence on the bubbly region; the final dimension of meshes are set to $10 \mathrm{~cm}$ in the radial direction and $39 \mathrm{~cm}$ in the height for the vessel. Three 2D-axisymmetric meshes with increasing refinement level have been used; the number of cells is respectively 1287, 5018 and 19812. We considered a two-phase, isothermal and turbulent flow with constant densities and viscosities. The LIM (Section 2.2) takes into account the fact that the interfacial friction effects are different in the large interfaces regions which are three cells thick and outside of these regions, where other two-phase models have to be used. In the present case, the bubbles induced effects in the bubbly region beneath the surface are taken into account via the corresponding momentum transfers (drag, added mass, turbulent dispersion and lift) which depend on the mean local bubble diameter, taken equal to its experimental value, $4 \mathrm{~mm}$.

\subsection{Results}

Each simulation has been performed in two steps: the first one corresponds to the transient establishment of the bubbly plume. The Fig. 6 shows the evolution of the void fraction in the jet region. The stabilization is reached within 10 seconds. In a second step, we performed temporal mean during 10 extra physical seconds.

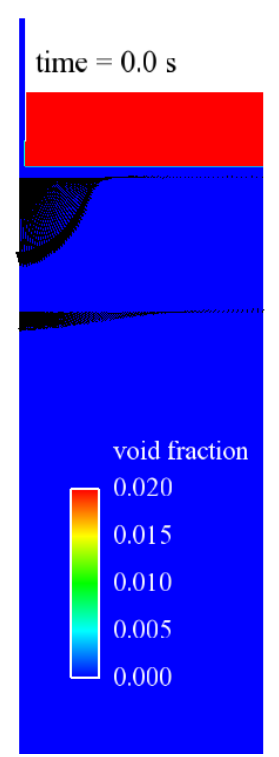

Fig. 6 : NCFD calculations of Igushi et al. experiment. Void fraction and liquid velocity profiles

during the transient simulation.
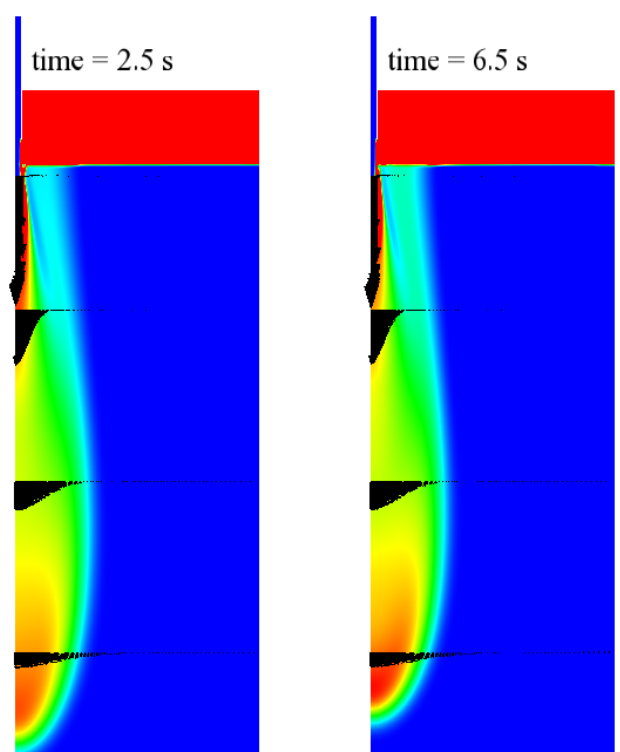
The Fig. 7 shows a comparison between experiment and numerical simulations; probes are located on the vertical central axis of the geometry at different distance from the free surface. For all graphs, square symbols denote experimental data and colored lines represent simulations performed with the three meshes (black: coarse, red: intermediate, green: finest mesh). The left (resp. top right) graph compares the mean (resp. rms) axial liquid velocity. This figure shows that the simulations are in good agreement with the experimental data, even if turbulence is slightly under-estimated. An additional single-phase jet simulation (light blue line) has been performed, in order to show the bubble effect on the jet expansion: the two-phase jet intensity (axial velocity) decreases faster than the single-phase one, because of bubbles rising up. The void fraction profile is presented on the bottom right; less than $3 \%$ of gas is entrained in the vessel.

Eventually we can say that the agreement and the weak sensitivity to the mesh refinement are very satisfactory and that the numerical results are very encouraging even using the coarse mesh. The results are improved in comparison to similar CFD by (Galassi et al., 2008) in the sense that in (Galassi et al., 2008) the void fraction prediction always seemed to change without coherence considering the different grids.
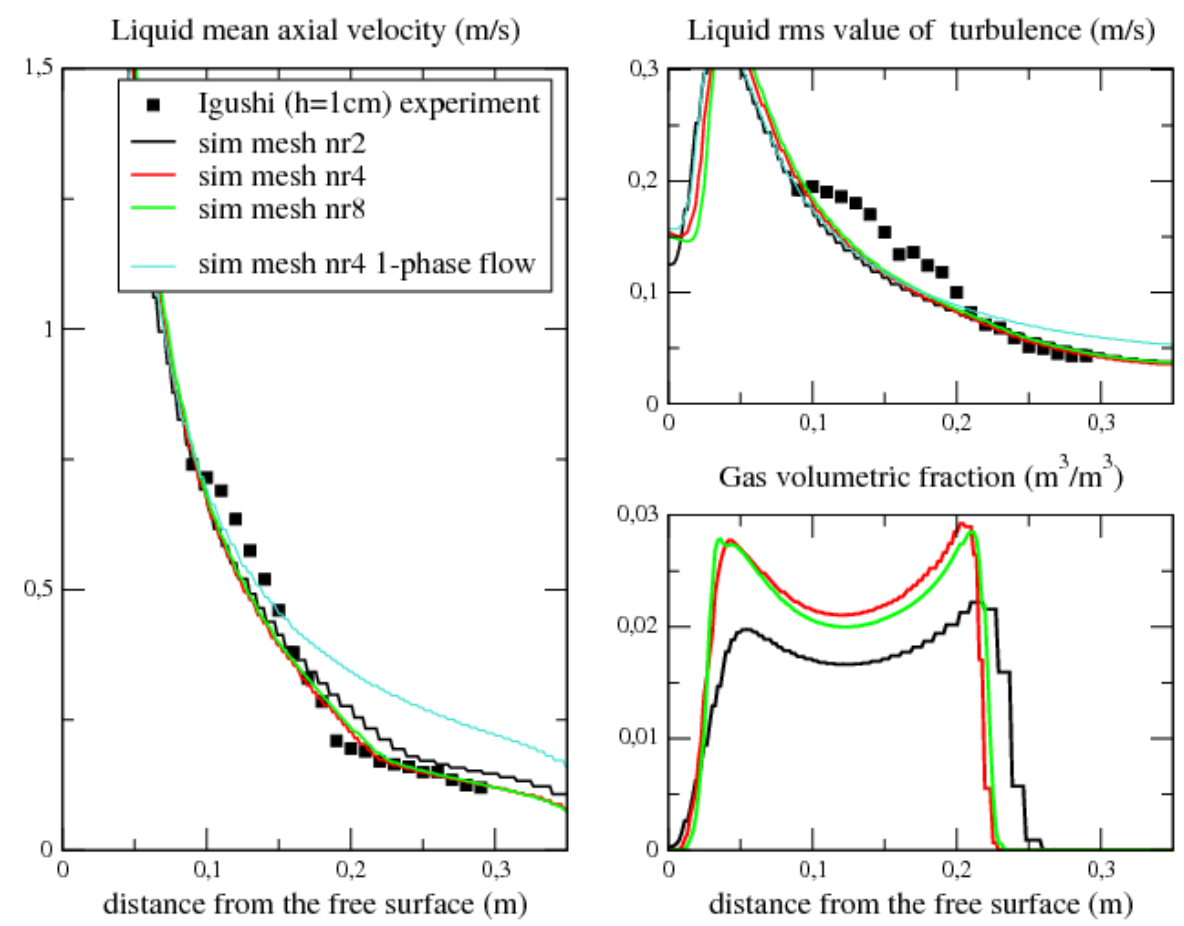

Fig. 7 : Liquid mean (left) and rms (top right) velocity, gas void fraction (lower right) on the vertical central axis of the domain, function of the distance to the free surface, comparison between experimental data (symbols) and NCFD simulations (continuous lines). 


\section{COSI: PTS VALIDATION IN REACTOR CONDITIONS}

\subsection{Description of the test case}

The COSI experiment was carried out at the CEA Grenoble for the purpose of studying complex condensation phenomena that take place in the area of the ECC injection during a loss of coolant accident in a PWR. COSI represents a 900 MW PWR cold leg with the safety injection at the scale of 1/100 in volume and conservation of Froude number (Janicot and Bestion, 1993). This reduced-scale experiment consists of a horizontal pipe that takes the place of the cold leg, an injection nozzle located at the center of the test section and a downcomer represented by a simple cylinder. Steam is supplied to one of the extremities of the test section, the other being partially opened or closed, depending on the runs. As shown on Fig. 8, there can be a weir downstream of the cold branch before the downcomer, in order to control the water level.

COSI covers LOCA conditions with many steady-state and transient tests. For the present NCFD validation, we focus attention on three steady-state runs since such runs allow a better evaluation of the experimental condensation rate: one with a 0.6 diameter high weir (run 11.6) and two others without weir which differ from the liquid and steam flows (runs 02.5 and 03.8 ).
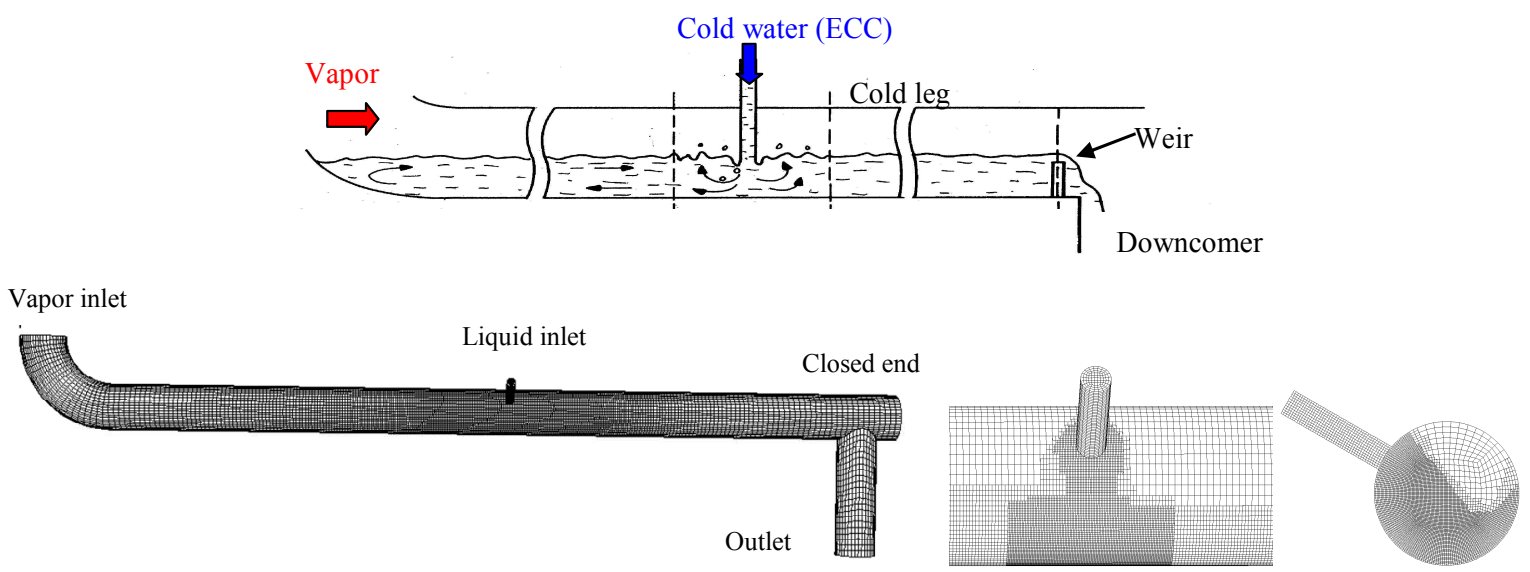

Fig. 8 : Top: schematic view of COSI experiment in the case with a weir. Bottom: coarser mesh for run 11.6 on the left and details of the most refined mesh for the run 02.5 on the right (m025_3, refined twice).

\subsection{Computational representation}

The runs have been investigated with two or three meshes in order to evaluate the mesh sensitivity. The coarser hexahedral meshes are created by SALOME (2010) by merging pieces of mesh, each piece being meshed with an O-grid approach. The refined meshes for run 11.6 were done in the same way by just increasing cells number (170 000 for m116_1, 280 for m116_2 and 800000 for m116_3). For runs 02.5 and 03.8 , the refined meshes are deduced from the first one by a refining technique using the software HOMARD (2010): only the interesting regions of the domain are refined according to some criteria defined by the user. In this case, what is interesting is the liquid temperature which is mainly controlled by condensation which depends strongly on the heat tranfer from the liquid to the interface. Therefore the liquid region is refined, which makes the resulting mesh depend on the run. Moreover, an important part of the condensation takes place in the ECC region, so a further refinement is made there..

\subsection{Results}

The measurements include temperature profiles at eight sections of the cold leg. A global condensation rate in the whole section is deduced from liquid and steam flows. The Fig. 9 compares 
experimental temperature profiles at six locations with those obtained by NCFD with the different meshes.

In the run 11.6, the jet is under the free surface and there is therefore no direct condensation between jet and steam. In the vicinity of the injection, the liquid temperature profile is quite well predicted. Nevertheless, the liquid remains too cold both upstream and downstream of the jet. There is not enough heat and momentum exchange between the higher part and the lower part of the stratified flow. The water height is quite well predicted upstream but under-estimated downstream.

In the runs 02.5 and 03.8 , the water height is quite well predicted, except near the steam inlet for 02.5 or towards the outlet for 03.8 . In both runs, the calculated water height is lower than the measured one just after the jet. The predicted temperatures are generally warmer than the experimental values. The condensation occurs especially in the vicinity of the jet. In these calculations without weir, the amount of vapor condensed in the region of the downcomer -which computational representation is presently defective- is less than $10 \%$ of the vapor condensed in the whole domain. This calculated condensed vapor in the whole domain compares to the measured one as shown in Table 2. Thus, the condensation rate increases with the mesh refinement in the run 02.5 , even if the temperature profiles do not depend strongly on the mesh refinement. In the run 03.5 , the condensation rate increases only slightly with the mesh refinement. In the run 11.6, the temperature profiles do not seem to depend on mesh refinement. Contrary to the results obtained with previous NCFD releases, the mesh sensitivity is finally quite moderate concerning the global condensation rate and the liquid temperature.

\begin{tabular}{|l|l|l|l|}
\hline Case & Rough mesh (_1) & Medium mesh (_2) & Fine mesh (_3) \\
\hline $\mathbf{0 2 . 5}$ & $-24 \%$ & $-18 \%$ & $-14 \%$ \\
\hline $\mathbf{0 3 . 8}$ & $-1 \%$ & $+2 \%$ & \\
\hline
\end{tabular}

Table 2 : NCFD calculations of COSI, discrepancies on the condensation rate in the whole domain.
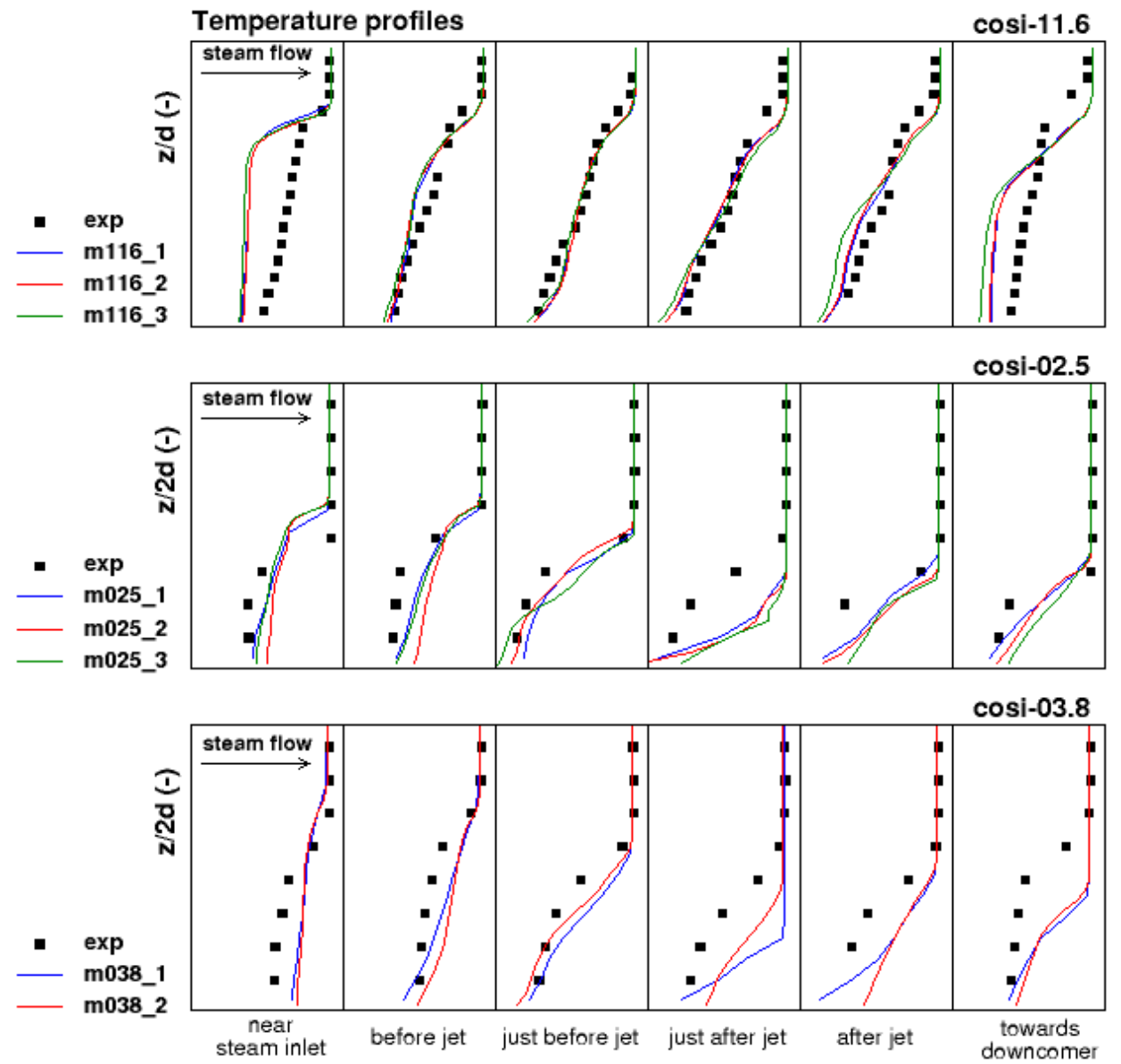

Fig. 9 Liquid temperature profiles at 6 locations of the test section. Comparison between experimental date (symbol) and NCFD simulations with various meshes (continuous lines). 


\section{CONCLUSION}

The validation for PTS of NEPTUNE_CFD, a two-phase CFD code, is presented in this paper. Five experiments were selected for that, after a process of phenomena identification, ranking table and state of the art review: stratified air-water and steam-water flows, liquid jets impinging a free surface, global validation with COSI. All calculations are done with the same set of models which includes the Large Interface Method (LIM) and a RANS approach with $(k-\mathcal{E})$ transport equations in each phase.

The BPG's recommend to include experimental uncertainties in the analysis. The available data about it in the cases considered in this paper lead to uncertainties which are much smaller than the typical discrepancies between our two-phase CFD calculations and measurements. Therefore this point is actually not a concern at the present stage in the present test cases.

The main limitations of the present two-phase CFD can be drawn. In fully developed channel airwater wavy stratified flow, the important phenomena of turbulence anisotropy and possibly the interaction between the mean liquid current flow and waves are not modeled which prevents us to have as satisfactory results as tools especially designed for this kind of flow. In steam-water stratified flow, results are still too much grid-dependent. In liquid jet impinging free surfaces: at low entrained volume fraction, the results are satisfactory; at high entrained volume fractions, the prediction of the gas entrainment at the jet impact on the free surface remains problematic.

Following the BPG's, the mesh sensitivity is investigated. The five experiments all deal with free surfaces. In this case, the BPG's concede that it is not possible to obtain completely grid-independent results and this is actually what we found. The expert can judge whether it is acceptable or not for a given purpose. This grid dependency is different from one test case to another and it turns out that in the most global experiment -the closest to the PTS industrial application- (COSI) it is rather weak. We conclude that the two-phase CFD with the LIM can be used as a tool for PTS investigations keeping in mind those limitations of the approach which are sketched by the present test cases and which stimulate further studies.

\section{ACKNOWLEDGMENTS}

The authors are grateful to Marc Boucker (EDF), Alain Martin (EDF) and Dominique Bestion (CEA) for support and discussions. The NEPTUNE project is funded by EDF (Electricité de France), CEA (Commissariat à l'Energie Atomique), AREVA-NP and IRSN (Institut de Radioprotection et de Sûreté Nucléaire). This work is also financially supported by the NURISP research project of the Euratom 7th Framework Programme (GA n $\left.{ }^{\circ} 232124\right)$.

\section{REFERENCES}

S. Banerjee, "A surface renewal model for interfacial heat and mass transfer in transient two-phase flow", Int. J. Multiphase Flow, 4, 571-573 (1978).

S. Banerjee et al., "Surface divergence models between turbulent streams", Int. J. Multiphase Flow, 30, 963-977 (2004).

S.G. Bankoff, "Some condensation studies pertinent to LWR safety", Int. J. Multiphase Flow, 6, 51-67 (1980).

P.A. Berthelsen, T. Ytrehus, "Calculations of Stratified Wavy Two-Phase Flow in Pipes", Int. J. Multiphase Flow, 31, pp. 571-592, 2005.

F. Bonetto, R. T. Lahey,. An experimental study on air carry-under due to a plunging liquid jet, Int. J. Multiphase Flow, 19, 281-294 (1993).

P. Coste, "Computational simulation of multi-D liquid-vapor thermal shock with condensation", Proc. ICMF'04, Yokohama, Japan, May 30- June 4 (2004).

P. Coste et al., "Modeling turbulence and friction around a large interface in a three-dimension two-velocity eulerian code", Proc. NURETH-12, Pittsburgh, USA, September 30- October 4 (2007).

P. Coste et al., "A two-phase CFD approach to the PTS problem evaluated on COSI experiment", Proc. ICONE 16, Orlando, USA, May 11-15 (2008). 
P. Coste, J. Laviéville, "A Wall Function-Like Approach for-Two-Phase CFD Condensation Modeling of the Pressurized Thermal Shock", Proc. NURETH-13, Kanazawa, Japan, September 27October 2 (2009).

L. Davoust et al., "Air entrainment by a plunging jet: the dynamical roughness concept and its estimation by a light absorption technique", Int. J. Multiphase Flow, 28, 1541-1564 (2002).

J. Fabre et al., "Stratified Flow, Part I: Local Structure", Multiphase Science and Technology, 3, 285-301 (1987).

M.C. Galassi et al., "Two-Phase Flow Simulations for PTS Investigations by Means of NEPTUNE_CFD Code", Science and Technology of Nuclear Installations, vol. 2009, Article ID 950536, 12 p., Hindawi Publishing Corporation (2009).

A. Guelfi et al., "NEPTUNE - A New Software Platform for Advanced Nuclear ThermalHydraulics", Nuclear Science and Engineering, 156, pp. 281-324 (2007).

HOMARD : web site: http://www.code-aster.org/outils/homard (2010).

M. Igushi et al., "Mean Velocity and Turbulence Characteristics of Water Flow in the bubble Dispersion Region induced by Plunging Water Jet", Int. J. Multiphase Flow., Vol. 24, No.4, 523-537 (1998).

M. Ishii, "Thermo-fluid Dynamics Theory of Two-Phase Flow", Eyrolles, Paris (1975).

A. Janicot, D. Bestion, "Condensation modeling for ECC injection", Nuclear Engineering and Design, 145, 37-45 (1993).

D. Lakehal et al., "Direct numerical simulation of condensing stratified flow", ASME J. Heat Transfer, 130, 021501 (2007).

J. Laviéville, P. Coste, "Numerical modeling of liquid-gas stratified flows using two-phase Eulerian approach", Proc. 5th International Symposium on Finite Volumes for Complex Applications, Aussois, France, June 08-13 (2008).

I.S. Lim et al., "Condensation measurement of horizontal cocurrent steam-water flow", J. Heat Transfer, 106, 425 (1984).

D. Lucas et al., "An Overview of the Pressurized Thermal Shock Issue in the Context of the NURESIM Project", Science and Technology of Nuclear Installations, Vol. 2009, 13 p., Hindawi Publishing Corporation (2009).

J. Magnaudet, I. Calmet, "Turbulent mass transfer through a flat shear-free surface", J. Fluid Mech., 553, 155-185 (2006).

N. Méchitoua et al., "An unstructured finite volume solver for two-phase water/vapor flows modeling based on an elliptic-oriented fractional step method", Proc. NURETH-10, Seoul, Korea, 5-9 October (2003).

F. Meknassi et al., "Numerical Modeling of Wavy Stratified Two-Phase Flow in Pipes", Chem. Eng. Sc., 55, pp. 4681-4697 (2000).

S. Pigny, P. Coste, "Simulation and Modeling of Two-Phase Bubble Flows", Proc. NURETH-11, Avignon, France, October 2-6 (2005).

SALOME: web site : http://www.salome-platform.org (2010).

M. Schmidkte, D. Lucas (2009), "CFD Approaches for Modeling Bubble Entrainment by an Impinging Jet", Science and Technology of Nuclear Installations, Vol. 2009, 12 p., Hindawi Publishing Corporation (2009).

T.G. Theofanous et al., "Turbulent mass transfer at free, gas-liquid interfaces, with applications to open-channel, bubble and jet flows", Int. J. Heat Mass Transfer, 19, 613-624 (1976).

W. Yao et al., "A three-dimensional two-fluid modeling of stratified flow with condensation for pressurized thermal shock investigations", J. Nuclear Technology, 152, 129-142 (2005). 